\title{
Matrix Method for Modelling of Multicomponent and Multistream Energy Systems and Installations of Thermal Power Plants
}

\author{
Barochkin A.E. \\ Ivanovo State Power Engineering University \\ Ivanovo, Russian Federation
}

\begin{abstract}
The aim of this work is to increase the operational efficiency of the multicomponent multithreaded power units and systems of the TPP using modeling, calculation and optimization. The goal is achieved by solving the following tasks: development of the tasks' classification system and a unified methodology for the mathematical description of energy formation and mass flows' processes in multicomponent and multithreaded power units of the TPP; development of a model of a steam turbine power unit; development of a model of heat and mass transfer processes in multi-stage multistream multiphase systems. The most significant results obtained were: the developed unified methodology for the matrix description of the processes of energy and mass flows' formation in multicomponent multistream energy systems of the TPP. Within the framework of the proposed methodology, a model of a steam turbine power was developed; model solutions were obtained and analyzed in order to calculate the energy characteristics of a heating turbine unit, the reliability and validity of the proposed approach was shown, a mathematical model of multistream multi-stage heat exchange systems were developed. The significance of the results obtained consisted in the development of a simple but informative mathematical model of a thermal power plant turbine generator and a model of multistream multi-stage heat exchange systems, each stage of which can have an arbitrary number of input and output flows with a possible phase transition in heat carriers.

Keywords: matrix methodology of modeling, phase transition, classification of problems, contact apparatus, multicomponent multistream systems, inverse problem, numerical experiment.

DOI: https://doi.org/10.52254/1857-0070.2021.4-52.06

UDC: 621.165
\end{abstract}

Metoda matriceală la modelarea sistemelor și instalațiilor energetice cu multe fluxuri și multe componente la centralele termoelectrice

Barocikin A.E.

Universitatea de Stat de Energetică din Ivanovo

Ivanovo, Federația Rusă

Rezumat. Scopul lucrării este de a crește eficiența funcționării centralelor termoelectrice (CTE) și a sistemelor cu multe fluxuri și multe componente prin modelarea, calculul și optimizarea acestora. Acest obiectiv este atins prin soluționarea următoarelor sarcini: elaborarea unui sistem de clasificare a sarcinilor și a unei metodologii unificate pentru descrierea matematică a proceselor de formare a fluxurilor de energie și masă în instalațiile energetice cu multe fluxuri şi multe componente a CTE, elaborarea, în cadrul metodologiei propuse, a unui model de instalație cu turbină cu abur și a unei abordări unificate pentru a descrie o CTE ca un sistem energetic cu multe fluxuri; elaborarea unui model de procese de transfer de căldură și masă în sisteme multi-fazice multiflux cu mai multe trepte, fiecare treaptă poate avea un număr arbitrar de fluxuri de intrare și de ieșire. Cele mai semnificative rezultate științifice sunt: elaborarea metodologiei unificată pentru descrierea matriceală a proceselor de formare a fluxurilor de energie și masă în sisteme energetice multicomponente şi cu multe fluxuri și instalații CTE. Importanța rezultatelor obținute constă în elaborarea unui model matematic simplu, dar informativ, al unui turbogenerator CTE şi a unui model al sistemelor de schimb de căldură cu mai multe fluxuri în mai multe trepte, unde fiecare treaptă poate avea un număr arbitrar de fluxuri de intrare și de ieșire cu un posibil tranziţie de fază în agenții termici, disponibile pentru utilizare directă în practica inginerească. Algoritmul propus de construcție a modelului poate fi aplicat eficient la actualizarea caracteristicilor energetice a turbogeneratoarelor și la soluționarea sarcinilor de proiectare a sistemelor de transfer de masă și căldură și a aparatelor de contact.

Cuvinte-cheie: metodologie, model matriceal, clasificare, codificare, instalație cu turbină cu abur, tranziţie de fază, sisteme cu multe fluxuri și multe componente, experiment numeric.

(C) Барочкин А. Е., 2021 


\section{Матричный метод при моделировании многокомпонентных и многопоточных энергетических систем и установок ТЭС \\ Барочкин А.E.}

Ивановский государственный энергетический университет

Иваново, Российская Федерация

Аннотация. Целью работы является повышение эффективности функционирования многокомпонентных многопоточных энергетических установок и систем ТЭС путем их моделирования, расчета и оптимизации. Поставленная цель достигается путем решения следующих задач: разработка системы классификации задач и единой методологии математического описания процессов формирования энерго- и массопотоков в многокомпонентных и многопоточных энергетических установках ТЭС; разработка в рамках предложенной методологии модели паротурбинной установки и единого подхода к описанию ТЭС как многопоточной энергетической системы; разработка модели процессов тепломассообмена в многоступенчатых многопоточных многофазных системах, каждая ступень которых может иметь произвольное число входных и выходных потоков. Наиболее существенными научными результатами являются: разработана единая методология матричного описания процессов формирования энерго-и массопотоков в многокомпонентных многопоточных энергетических системах и установках ТЭС. В рамках предложенной методологии разработаны модель паротурбинной установки и единый подход к математическому описанию ТЭС как многопоточной энергетической системы; получены и проанализированы решения модели с целью построения энергетических характеристик теплофикационного турбоагрегата, выполнено сравнение результатов расчета с энергетическими характеристиками действующего турбоагрегата, показана достоверность и обоснованность предложенного подхода. Разработана математическая модель многопоточных многоступенчатых теплообменных систем, каждая ступень которых может иметь произвольное число входных и выходных потоков. Значимость полученных результатов состоит в разработке простой, но информативной математической модели турбогенератора ТЭС и модели многопоточных многоступенчатых теплообменных систем, каждая ступень которых может иметь произвольное число входных и выходных потоков с возможным фазовым переходом в теплоносителях, доступной для непосредственного использования в инженерной практике. Предложенный алгоритм построения модели может эффективно применяться при актуализации энергетических характеристик турбоагрегатов и решении задач проектирования тепломассообменных систем и контактных аппаратов.

Ключевые слова: методология, матричная модель, классификация, кодификация, паротурбинная установка, фазовый переход, многокомпонентные многопоточные системы, численный эксперимент.

\section{ВВЕДЕНИЕ}

Традиционно задачи теплопередачи решаются применительно к двухпоточным системам, в которых теплообмен осуществляется между горячим и холодным теплоносителями [1-6]. Однако наряду с двухпоточными системами нередко встречаются многопоточные системы, в которых число потоков теплоносителей составляет три и более [7-9]. Кроме этого каждый поток энергоносителей может состоять из нескольких компонентов. В качестве компонентов понимаются различные фазовые состояния одного вещества (например, вода и пар) или разные компоненты смеси, отличающиеся физическими или химическими свойствами (крупностью зерен для сыпучих материалов или разными температурами кипения смеси жидкостей) [10-18].

Несмотря на большое количество работ, посвященных моделированию процессов тепломассобмена [19-23], в них не рассматривается многокомпонентность теплоносителей.

В энергетике, в пищевой и нефтехимической отраслях промышленности, часто в тепломассообменных процессах, участвуют многокомпонентные теплоносители, теплофизи- ческие свойства компонентов которых существенно различаются. Для проведения теплотехнических расчетов с такими смесями обычно выполняется усреднение значений теплофизических параметров компонентов. Например, воздух при проведении тепловых расчетов считается газом со своей плотностью и теплоемкостью.

Однако в ряде технологий на основе различая теплофизических свойств, в частности, различия температуры кипения компонентов реализуются процессы по разделению этих компонентов, например, при перегонке или ректификации продуктов в пищевой и нефтехимической промышленности. В этом случае именно различия в теплофизических свойствах каждого компонента необходимо учитывать при расчете тепломассообменных процессов.

Наконец вся тепловая схема ТЭС может рассматриваться как многопоточная система с обменом между ее подсистемами потоками разного вида энергии: химической, тепловой, механической, электрической. Развитие и совершенствование энергосберегающих технологий в современных теплообменных систе- 
мах, включающих большое число подсистем и связей между ними, во многом сдерживается отсутствием методов расчета и современных компьютерных комплексов, позволяющих адекватно прогнозировать состояние таких систем во всем диапазоне нагрузок оборудования.

Представление на единой методологической основе трансформации энергии на тепловой станции открывает перспективу построения актуальных энергетических характеристик оборудования при использовании ограниченного объема экспериментальных данных.

Кроме этого единый подход к моделированию процессов трансформации энергии позволяет проводить оптимизацию функционирования оборудования.

Таким образом, развитие методов моделирования многопоточных теплообменных аппаратов на случай описания многокомпонентных многопоточных теплообменников является актуальной задачей для энергетической и смежных отраслей промышленности.

\section{МЕТОДЫ, РЕЗУЛЬТАТЫ И ОБСУЖДЕНИЕ}

Для систематизации существующих задач и моделей тепломассообменных процессов проведен анализ опубликованных данных [1, 3-29]. Классификация задач выполнена по четырем признакам: число компонентов, число потоков, число ступеней в анализируемой системе, наличие возможного перехода между компонентами. По числу компонентов выделяются однокомпонентные и многокомпонентные системы. По числу потоков теплоносителей системы подразделяются на однопоточные, двухпоточные и многопоточные.

По признаку числа ступеней системы классифицируются на одноступенчатые и многоступенчатые. К многоступенчатым [3] будем относить системы, которые включают две и более ступеней. Кроме этого при классификации задач рассматривается признак возможного перехода одного компонента в другой, что на практике может быть реализовано при фазовых переходах или при переходе крупных фракций в мелкие при измельчении сыпучего материала. Дополнительно для тепломассообменных систем предложена система их кодификации. Система кодификации построена следующим образом: код состоит из чисел, разделенных точками: первое слева число кода показывает количество компонен- тов, второе - количество потоков, третье количество ступеней, а четвертое - наличие (1) или отсутствие (0) возможного перехода между компонентами. Например, код задачи 1.1.1.0 показывает, что моделируемая система однокомпонентная, однопоточная, одноступенчатая при отсутствии возможных переходов между компонентами. Ранее [3] рассмотрены матричные модели для однокомпонентных двухпоточных одноступенчатых систем (код задачи 1.2.1.0) и для многоступенчатых (m ступеней) двухпоточных систем (код задачи 1.2.m.0). Для однокомпонентных трехпоточных одноступенчатых и многоступенчатых, систем код задачи представляется в виде 1.3.1.0 и 1.3.m.0 соответственно.

Предложенная система классификации и кодификации позволяет более четко определить тип задач и ориентироваться в степени ее изученности.

Предлагаемая методология для разработки математических моделей технологических систем основана на использовании матричного подхода, который хорошо себя зарекомендовал при решении целого ряда научных и практических задач [3]. Следует отметить, что данный подход целесообразно использовать для описания систем, которые могут быть охарактеризованы аддитивными определяющими систему параметрами, для которых могут быть записаны балансовые соотношения в виде закона сохранения.

Суть методологии и алгоритм построения математической модели в рамках этой методологии рассмотрим на ряде примеров новых задач или известных задач, сформулированных для новых условий или новых объектов.

В качестве первого примера рассматривается описание паротурбинной установки и единого подхода к математическому описанию ТЭС с целью построения энергетических характеристик теплофикационного турбоагрегата типа ПТ с производственным и теплофикационным отборами пара. Модель строится для многопоточной многокомпонентной энергетической системы с возможной трансформацией одного вида энергии в другой.

Принципиальная тепловая схема паротурбинной установки представлена на рис. 1,a. Топливо (F) поступает в котел (SB), где при сжигании химическая энергия топлива преобразуется в тепловую энергию пара. Пар из котла подается в паровую турбину (ST), где тепловая энергия пара трансформируется в 
механическую энергию вращения лопаток ротора турбины.

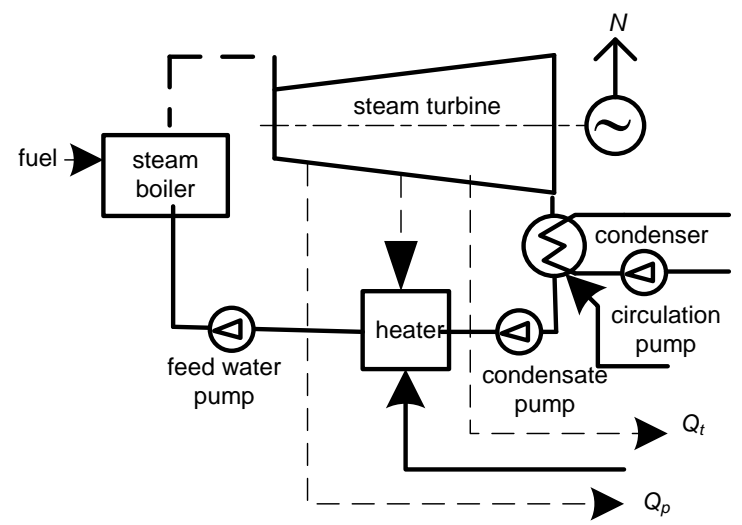

a)

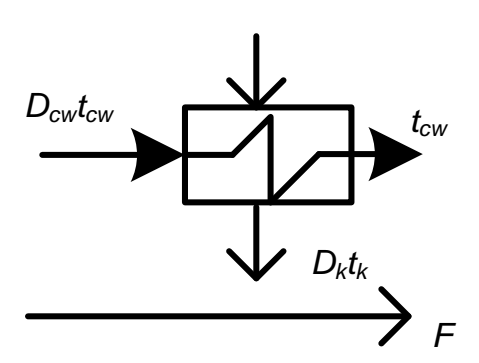

b)

Рис.1. Принципиальная тепловая схема паротурбинной установки типа ПТ (а) и расчетная схема модели конденсатора (b).

Fig.1. A schematic thermal diagram of a PT type turbine unit (a) and a design diagram of a condenser model (b).

В турбогенераторе энергия вращения преобразуется в электрическую энергию. В конденсаторе (С) происходит конденсация отработавшего в турбине пара. Образовавшийся конденсат пара подается конденсатным насосом (Р) в регенеративный подогреватель $(\mathrm{H})$, в котором происходит его нагрев паром, поступающим из нерегулируемого отбора турбины. Система регенеративного подогрева питательной воды представлена на рис. 1,a только одним смешивающим подогревателем. После регенеративного подогрева питательная вода прокачивается питательным насосом (FP) в паровой котел. Из регулируемых отборов турбины пар направляется на производственные нужды (тепловая мощность $Q_{p}$ ) и теплофикацию (тепловая мощность $Q_{t}$ ). В схеме учтен возврат конденсата пара производственного отбора в регенеративный подогреватель. Конденсат пара теплофикационного отбора возвращается в конденсатор.

Для разработки уточненной матричной модели с учетом принципиальной тепловой схемы рис. 1, а строится расчетная схема модели конденсатора, представленная на рис. 1,b. При конденсации пара в конденсаторе температура пара, которая и определяет конечное давление в конденсаторе, считается для выделенного режима постоянной $\left(t_{k}=\right.$ const $)$, но может варьироваться в зависимости от нагрузки. В качестве определяющей координаты процесса конденсации выбирается поверхность теплообмена $F$ (рис. 1,b). Для описания состояния пара при его конденсации используется степень сухости $x$, которая показывает массовую долю пара в пароводяной смеси. При фазовом переходе в горячем теплоносителе из баланса теплоты, составленного для холодного и горячего теплоносителей [3], получена система дифференциальных уравнений, описывающая изменение степени сухости горячего $(x)$ и температуры холодного $\left(t_{c w}\right)$ теплоносителей вдоль поверхности теплообмена.

$$
\left\{\begin{array}{l}
\frac{d x}{d F}=-a_{1}\left(t_{k}-t_{c w}\right) \\
\frac{d t_{c w}}{d F}=a_{2}\left(t_{k}-t_{c w}\right)
\end{array},\right.
$$

где $a_{1}=k / r D_{k} ; a_{2}=k / c D_{u в} ; F-$ поверхность нагрева; $x$ - степень сухости пара; $k$ - коэффициент теплопередачи; $r$-удельная теплота парообразования, $\boldsymbol{c}$-удельная теплоемкость; $D_{k}-$ расход пара в конденсатор; $D_{u s}-$ расход циркуляционной воды.

Для заданных начальных условий: $F=O$, $x=x_{0}, t_{c w}=t_{c w 0}$, решение системы (1) относительно температуры охлаждающей воды принимает вид

$$
t_{u s}=t_{k}-\left(t_{k}-t_{u s 0}\right) \exp \left(-a_{2} F\right)
$$

Приравнивая теплоту нагрева воды $D_{c w} c\left(t_{c w}-t_{c w 0}\right)$ теплоте конденсации пара $D_{k}\left(i_{k}-i_{k s}\right)$, записывается баланс энергии

$$
D_{k}\left(i_{k}-i_{k s}\right)=D_{c w} c\left(t_{k}-t_{c w 0}\right)\left(1-\exp \left(-a_{2} F\right)\right),(2)
$$

где $i_{k}, i_{k s}$-энтальпия пара и конденсата.

Ранее при разработке базовой модели турбоустановки сделаны следующие упрощения и допущения: один котел снабжает паром одну турбоустановку; котел представляет собой рекуперативный теплообменник, в котором вода нагревается, испаряется, а пар перегре- 
вается; КПД брутто котла считаем известным; система регенеративного подогрева питательной воды включает один смешивающий подогреватель.

При разработке модели согласно расчетной схеме рис. 1 , а записываются следующие балансовые уравнения: баланс энергии для котла; баланс энергии для турбины; баланс массы для турбины; баланс энергии для регенеративного подогревателя; баланс энергии для конденсатора; баланс энергии для производственного отбора пара; баланс энергии для теплофикационного отбора пара. Подробно эти уравнения рассмотрены ранее при разработке базовой модели. Принципиальное отличие предлагаемой модели заключается в

$$
A=\left[\begin{array}{cccc}
Q \eta_{k} & -\left(i_{o}-i_{f v}\right) & 0 & 0 \\
0 & 0 & \left(i_{o}-i_{p}\right) \eta_{t} & \left(i_{o}-i_{r}\right) \eta_{t} \\
0 & i_{p v} & -i_{p s} & -i_{r} \\
0 & 1 & -1 & -1 \\
0 & 0 & 0 & 0 \\
0 & 0 & i_{p}-i_{p s} & 0 \\
0 & 0 & 0 & 0
\end{array}\right.
$$

здесь $X$ - матрица-столбец искомых значений; $X_{v}$ - матрица-столбец заданных величин; верхний индекс «'» означает транспонирование матрицы; $A$ - матрица коэффициентов; $D$-расход теплоносителя; $i$ - энтальпия; $N$ - электрическая мощность; $B$ - расход топлива; $Q$ - удельная теплота сгорания топлива; $Q_{p}, Q_{t}-$ производственная и теплофикационная нагрузки; $\eta_{t}$ - произведение внутреннего относительного КПД проточной части турбины на электромеханический КПД турбоустановки; $\eta_{k}$ - произведение КПД брутто котла на КПД теплового потока (учитывает потери теплоты при транспорте пара от котла до турбоустановки и питательной воды от турбоустановки до котла); индекс «о» относится к острому пару; «р» - производственному отбору; «t» - теплофикационному отбору; «r» - регенеративному отбору; «cw»циркуляционному водоснабжению; «k» конденсатору; «s» - состоянию насыщения; «fw»- питательной воде.

Решение системы (3), выполненное методом обращения матриц, позволяет определить вектор искомых параметров согласно выражению введении в нее уравнения (2) для описания баланса энергии в конденсаторе.

Перечисленные выше семь балансовых соотношений с учетом (2) записываются в матричном виде

$$
A X=X_{v},
$$

где

$$
X=\left[\begin{array}{lllllll}
B & D & D_{p} & D_{r} & D_{t} & D_{k} & t_{k}
\end{array}\right]^{\prime},
$$

$$
X_{v}=\left[\begin{array}{llllll}
0 & N & 0 & 0 & -D_{c w} c t_{c w 0}\left(1-\exp \left(-a_{2} F\right)\right) Q_{p} & Q_{t}
\end{array}\right]
$$

$$
\left.\begin{array}{ccc}
0 & 0 & 0 \\
\left(i_{o}-i_{t}\right) \eta_{t} & \left(i_{o}-i_{k}\right) \eta_{t} & 0 \\
-i_{k s} & -i_{k s} & 0 \\
-1 & -1 & 0 \\
0 & i_{k}-i_{k s} & -D_{c w} c\left(1-\exp \left(-a_{2} F\right)\right) \\
0 & 0 & 0 \\
i_{t}-i_{k s} & 0 & 0
\end{array}\right]
$$

$$
X=A^{(-1)} X_{v}
$$

где возведение матрицы $A$ в степень $(-1)$ означает ее обращение.

Для построения энергетических характеристик оборудования ТЭС $[2,3]$ определяется удельный расход тепловой энергии брутто на выработку электроэнергии $q_{t}$, ккал/(кВт·ч), который является величиной обратной абсолютному электрическому КПД турбоустановки $\eta[3]$ :

$$
\begin{gathered}
\eta=N /\left(B Q \eta_{k}-Q_{t}-Q_{p}\right) ; \\
q_{t}=3600 /(\eta \cdot 4,19)
\end{gathered}
$$

Адекватность разработанной модели (1-6) проверяется на примере расчета энергетической характеристики турбины ПТ-65/75130/13. Результаты расчетного анализа, проведенного в рамках предложенной модели (1-6), представлены на рис. 2 в виде интегральных энергетических характеристик турбоустановки

$$
Q=q_{t} N=f(N),
$$


где $Q$ - общий расход тепловой энергии брутто турбоустановки на выработку электроэнергии.

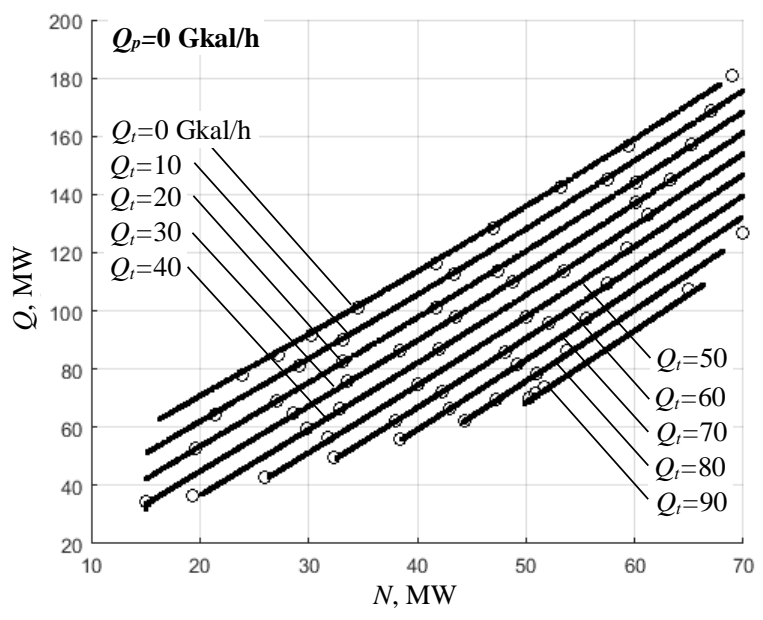

Рис.2. Энергетические характеристики турбины ПТ-65/75-130/13 для нагрузки производственного отбора $Q_{p}=0$ и теплофикационной нагрузки

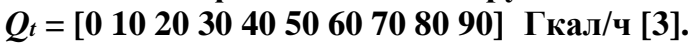

Fig.2. Energy characteristics of the turbine PT-65/75-130/13 for the production extraction load $Q_{p}=0$ and heating load values

$Q_{t}=\left[\begin{array}{llllllllll}0 & 10 & 20 & 30 & 40 & 50 & 60 & 70 & 80 & 90\end{array}\right] \mathrm{Gcal} / \mathrm{h}[3]$.

Общий расход тепловой энергии для различных значений теплофикационной нагрузки и вырабатываемой электрической мощности вычисляется через произведение удельного расхода $q_{t}$, определенного согласно (4-6), умноженного на выработку электроэнергии $N$.

Энергетическая характеристика на рис. 2 построена для режима работы турбины с отсутствием отпуска пара из производственного отбора ( $Q_{p}=0$ Гкал/ч) и переменным отпуском пара из теплофикационного отбора ( $Q_{t}=\left[\begin{array}{llllllllll}0 & 10 & 20 & 30 & 40 & 50 & 60 & 70 & 80 & 90\end{array}\right]$ Гкал/ч).

Точками на рис. 2 показаны значения, принятые для данной турбины по данным нормативно-технической документации по топливоиспользованию ТЭЦ, линиями - результаты расчета.

Анализ приведенных результатов моделирования паротурбинной установки в рамках предложенной методологии показал, что учет в модели системы модели подсистемы конденсатора значимо влияет на качество моделирования по сравнению с базовой моделью.

Следующий пример использования матричной методологии моделирования рассмат- ривается для тепломассообменных систем с произвольным числом входных и выходных потоков в каждую ступень.

Ранее получено решение задачи матричного описания многоступенчатых двухпоточных систем теплообменных аппаратов, каждая ступень которых представлена в виде четырехполюсника с двумя входными и двумя выходными потоками [3]. Однако на практике число потоков на входе и выходе аппарата может быть больше двух, так как помимо двух основных потоков холодного и горячего теплоносителей часто направляются дополнительные потоки, обусловленные дренированием, продувкой или аварийными режимами системы. Подача перечисленных потоков теплоносителей может производиться в разные точки теплообменного аппарата, что обусловливает разную эффективность анализируемых процессов.

Для разработки математического описания многопоточных многоступенчатых систем предлагается расчетная схема объекта исследования, представленная на рис. 3. На рисунке приведена обобщенная структура соединения $n$ ступеней системы, при которой возможно соединение любых входных и выходных потоков при произвольном их числе для каждой ступени. На рисунке схематично показано формирование потока на входе в $i$-й элемент (ступень) установки. Более детально на рисунке показана связь между первой и $i$ ой ступенью. Так на вход в первый элемент подаются потоки теплоносителей, которые характеризуются набором аддитивных параметров $\left[X_{0}\right]_{1}$, в качестве которых рассматриваются потоки массы или энергии. Входные параметры обозначены индексом «0» внутри квадратных скобок, индекс «1» за скобками указывает к номер ступени. Процессы тепломассобмена описываются матрицей процесса $B$. Выходные параметры ступени определяются уравнением $[X]_{1}=B_{1}\left[X_{0}\right]_{1}$. Для указания направления движения потоков после ступени формируется матрица коммутации $K_{i 1}$, элементы которой показывают доли потоков теплоносителей, подаваемых из первой ступени в $i$-ю. При этом матричное произведение $K_{i 1} B_{1}\left[X_{0}\right]_{1}$ определяет параметры потоков, подаваемых из первого элемента в $i$-й. Подача потоков в $i$-ю ступень может быть выполнена не только из первой ступени, но и из остальных ступеней системы, а также из внешних систем. 


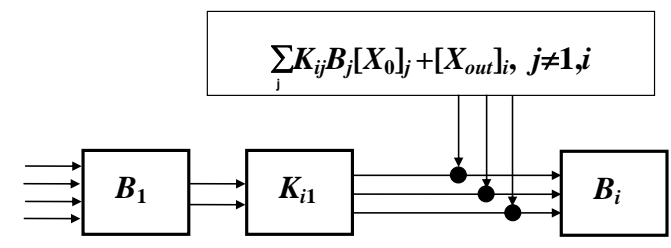

$\left[X_{0}\right]_{1} \rightarrow \quad B_{1}\left[X_{0}\right]_{1} \rightarrow K_{i 1} B_{1}\left[X_{0}\right]_{1} \rightarrow\left[X_{0}\right]_{i}$

Рис.3. Расчетная схема формирования параметров потока на входе в $\boldsymbol{i}$-й элемент многопоточной многоступенчатой теплообменной установки.

Fig.3. Calculation scheme for the formation of flow parameters at the input to the $i$-th element of a multi-flow multistage heat exchange plant.

Перечисленные потоки условно показаны в верхней части расчетной схемы на рис. 3 . Входной вектор признаков для $i$-го элемента определится суммой значений аддитивных параметров смешиваемых на его входе потоков в узлах смешения, отмеченных на рисунке точками:

$$
\left[X_{0}\right]_{i}=K_{i 1} B_{1}\left[X_{0}\right]_{1}+\ldots+K_{\text {in }} B_{n}\left[X_{0}\right]_{n}+\left[X_{\text {out }}\right]_{i}
$$

где индекс «out» указывает на внешний поток теплоносителя, подаваемый в теплообменный аппарат.

Уравнения, аналогичные (7), записываются для каждой из $n$ ступеней установки. В итоге формируется система уравнений

$$
\left(\begin{array}{cccc}
-I & K_{12} B_{2} & \cdots & K_{1 n} B_{n} \\
K_{21} B_{1} & -I & \cdots & K_{2 n} B_{n} \\
\cdots & \cdots & \cdots & \cdots \\
K_{n 1} B_{1} & K_{n 2} B_{2} & \cdots & -I
\end{array}\right)\left(\begin{array}{c}
{\left[X_{0}\right]_{1}} \\
{\left[X_{0}\right]_{2}} \\
\cdots \\
{\left[X_{0}\right]_{n}}
\end{array}\right)=\left(\begin{array}{c}
-\left[X_{\text {out }}\right]_{1} \\
-\left[X_{\text {out }}\right]_{2} \\
\cdots \\
-\left[X_{\text {out }}\right]_{n}
\end{array}\right)(8)
$$

где $I$ - единичная матрица.

Решение системы уравнений (8) позволяет найти значения параметров теплоносителей в любом элементе системы.

Вид матрицы процесса $B$ для описания теплообмена в двухпоточной ступени как с учетом, так и без учета фазового перехода в теплоносителях подробно рассматривается в работе [3].

Для иллюстрации возможности моделирования сложных многопоточных систем с помощью разработанного подхода, предлагается рассмотреть спиральный теплообменный аппарат, представленный на рис. 4. Сложность моделирования теплопередачи в спиральном теплообменном аппарате заключается в необходимости учета передачи тепловой энергии от анализируемого потока сразу двум соседним теплоносителям, расположенным внутри и снаружи анализируемого потока (рис. 4,b).

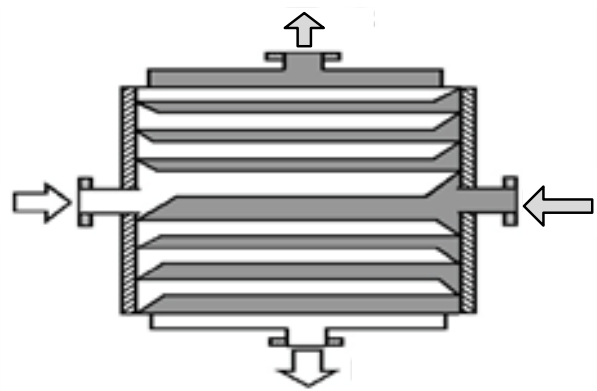

a)

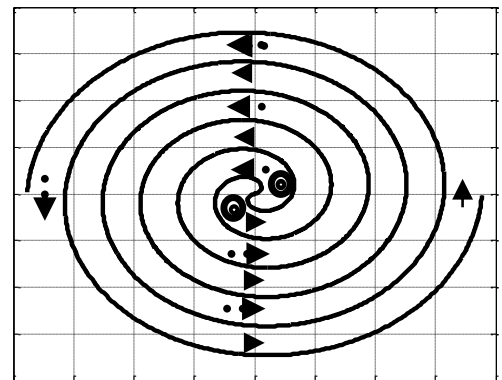

b)

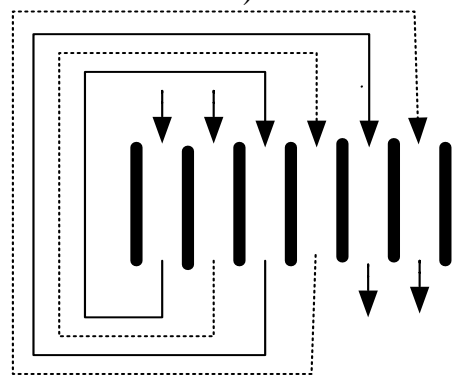

c)

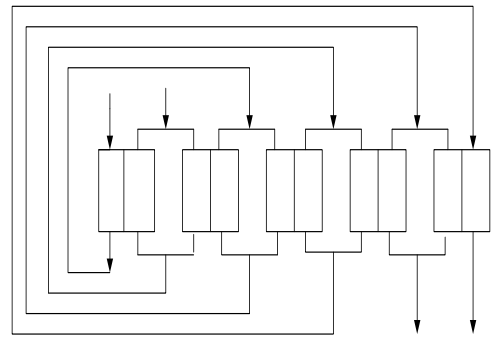

d)

Рис.4. Вид спирального теплообменного аппарата (a), схема потоков (b), модельное представление схемы потоков (c), эквивалентная расчетная структура потоков в виде четырехполюсников (d).

Fig.4. View of the spiral heat exchanger (a), flow diagram (b), model representation of the flow diagram (c), equivalent calculated flow structure in the form of four-port connections (d). 
Для использования матричной методологии для описания спирального теплообменного аппарата на рис. 4 приведен алгоритм приведения расчетной схемы теплообменника к виду, для которого можно применить уравнение (8) при построении матричных моделей.

Аппарат условно разрезается вдоль радиуса, разворачивается и представляется в виде эквивалентной схемы пластинчатого теплообменника на рис. 4,c. Ввиду разных радиусов разворачиваемых окружностей пластины эквивалентного теплообменника имеют разную площадь теплообмена.

\section{ВЫВОДЫ}

1. Разработана система классификации и кодификации задач и методология математического описания процессов формирования потоков энергии и массы в многокомпонентных и многопоточных энергетических установках ТЭС, позволяющая адекватно определять новизну и значимость решаемых задач для повышения эффективности функционирования энергетических систем;

2. Разработана в рамках предложенной методологии модель паротурбинной установки и единый подход к описанию ТЭС как многопоточной энергетической системы, показано, что учет в модели системы модели подсистемы конденсатора значимо влияет на адекватность модели во всем диапазоне нагрузок оборудования и позволяет актуализировать вид энергетических характеристик при ограниченном объеме экспериментальных данных;

3. Разработана математическая модель многопоточных многоступенчатых теплообменных систем, каждая ступень которых может иметь произвольное число входных и выходных потоков; что позволяет оптимизировать системы со сложной структурой связей между подсистемами по выбранной целевой функции.

4. Пример использования матричной методологии рассмотрен при моделировании спирального теплообменника, в котором каждый поток контактирует с двумя другими потоками теплоносителя через внутреннюю и внешнюю для него стенки, что обеспечивает возможность выбора оптимальных конструктивных и режимных параметров при проектировании и эксплуатации теплообменного оборудования.

\section{Литература (References)}

[1] Isachenko V.P., Osipova V.A., Sukomel A.S. Teploperedacha [Heat transfer]. - M.: Energoatom-Publishing House, 1981.

[2] NazmeevYu.G., Lavygin V.M. Teploobmennye Apparaty TES [Heat Exchangers of TPPCHP]. M.: Energoatom-Publishing House, 1981.

[3] Zhukov V.P., Barochkin E.V. Sistemnyi Analiz Energeticheskikh Teplomassoobmennykh Ustanovok [System Analysis of Energy Heat and Mass Exchange Plants]. Ivanovo: IGEU, 2009.

[4] Zarzycki R. Heat Exchange and Mass Transfer in Environmental Engineering. Warsaw, WNT, 2005.

[5] Rohsenow W.M., Hartnett J.P., Ganic J.P. Handbook of Heat Transfer Fundamentals, second ed.- New York:McGraw-Hill, 1985.

[6] Raghuraman J., Mohan V. Markov Chain Model for the Residence Time and Contact Time Distributions in Packed Beds. Chemical Engineering Science, 1975, vol. 30, pp. 549-553.

[7] Flavio K., Raquel Y. Experimental and Numerical Heat Transfer in a Plate Heat Exchanger. Chemical Engineering Science, 2006, vol. 61, pp. 7133-7138.

[8] Cave G.D., Giudici M., Pedrocchi E., Pesce G. Study of Fluid Flow Distribution Inside Plate Heat Exchangers by Thermographic Analysis. 1983.

[9] Cubillas P.R., Pérez-Lombard R. Transient Heat Conduction in Multi-Layer Walls: An efficient Strategy for Laplace's Method. Energy and Buildings, 2010, vol. 42. pp. 541-546.

[10] PFR Engineering Systems, Heat Transfer and Pressure Drop Characteristics of Dry Towers Extended Surfaces. Part II: DATA Analysis and Correlation. Calif. Marina del Rey: PFR, Rapport d'etude no. BNWL-BFR-7-102, 1976.

[11] Butterworth D. Condensation of vapor mixtures H.E.D.H. Handbook, Dusseldorf Hemisphere Publishing Corporation, 1983.

[12] Colburn A.P., Drew T.B. An Experimental Approach Based on Inverse Heat Conduction Analysis for Thermal Characterization of Phase Change Materials. Thermochimica Acta, 2020, vol. 685, March, 178540.

[13] Almeida A.P., Naveira-Cotta C.P., Cotta R.M. Transient Three-Dimensional Heat Conduction in Heterogeneous Media: Integral Transforms and Single Domain Formulation. International Communications in Heat and Mass Transfer, 2020, vol. 117, October, 104792.

[14] Marlin F. Condensation in the Presence of Uncondensable in the Environment of a Bundle of Tubes, Application to the Study of a Tube Exchanger. Grenoble, C.E.N. de Grenoble, S.T.T.L.P.M.L., Internship Report no. 88/07/ A, 1988. 
[15] Collier J.G. Convective Boiling and Condensation, Second ed., McGraw-Hill International Book Company Limited, Maidenhead, UK, 1981.

[16] Krishna R., Standart G.L. A Multicomponent Film Model Incorporating a General Matrix Method of Solution to the Maxwell-Stefan Equations, AIChE Journal. 1976, vol. 22, pp. 383-399.

[17] Tritscher P., Broadbridge P. A Similarity Solution of a Multiphase Stefan Problem Incorporating General Non-Linear Heat Conduction. International Journal of Heat and Mass Transfer, 1994, vol. 37, no. 14, pp. 2113-2121. https://doi.org/10.1016/0017-9310(94)90312-3.

[18] Agarwala S., Prabhu K.N. An Experimental Approach Based on Inverse Heat Conduction Analysis for Thermal Characterization of Phase Change Materials, Thermochimica Acta, 2020, vol. 685, March, 178540.

[19] Grossmann I.E., Yeomans H., Kravanja Z., 1998, A Rigorous Disjunctive Optimization Model for Simultaneous Flowsheet Optimization and Heat Integration, Computers \& Chemical Engineering, 22, 157-164.

[20] Hasan M.M.F., Karimi I.A., Alfadala H.E., Grootjans H., 2009, Operational Modeling of Multistream Heat Exchangers with Phase Changes, AIChE Journal, 55, 150-171.

[21] Kamath R.S., Biegler L.T., Grossmann I.E., 2012, Modeling Multistream Heat Exchangers with and without Phase Changes for Simultaneous Optimization and Heat Integration, AIChE Journal, 58, 190-204.

[22] Georgiadis, M.C., Macchietto, S., 2000. Dynamic Modelling and Simulation of Plate Heat Exchangers under Milk Fouling. Chemical Engineering Science, 55, pp.1605-1619.

[23] Nuijten A.D.W., Høyland K.V. Modelling the
Thermal Conductivity of a Melting Snow Layer on a Heated Pavement. Cold Regions Science and Technology, 2017, vol. 140, pp. 20-29. doi.org/10.1016/j.coldregions.2017.04.008

[24] Critoph R.E., Holland M.K, Fisher M. Comparison of Steady State and Transient Methods for Measurement of Local Heat Transfer in Plate Fin-Tube Heat Exchangers Using Liquid Crystal Thermography With Radiant Heating, International Journal of Heat and Mass Transfer, 1998, vol. 42 (1), pp.1-12.

[25] Ay H., Jang J.Y., Yeh J. Local Heat Transfer Measurements of Plate Finned-Tube Heat Exchangers by Infrared the Tomography, International Journal Heat Mass Transfer, 2002, vol. 45 (20), pp. 4069-4078.

[26] Li H., Kottke V. Visualization and Determination of Local Heat Transfer Coefficients in Shell-and-Tube Heat Exchangers for Staggered Tube Arrangement by Mass Transfer Measurements, Experimental Thermal and Fluid Science, 1998, vol. 17 (3), pp.210-216.

[27] Fernandes C.S., Dias R., Nybrega J.M., Afonso I.M., Melo L.F., Maia J.M. Simulation of Stirred Yoghurt Processing in Plate Heat Exchangers. Journal of Food Engineering, 2005, vol. 69. pp.281-290.

[28] Zhang B., Mei J., Zhang Y. A General Approach for Solving Three-Dimensional Transient Nonlinear Inverse Heat Conduction Problems in Irregular Complex Structures. International Journal of Heat and Mass Transfer, 2019, vol.140, pp. 909-917.

[29] Belghazi H., El Ganaoui M., Labbe J.C. Analytical Solution of Unsteady Heat Conduction in a Two-Layered Material in Imperfect Contact Subjected to a Moving Heat Source. International Journal of Thermal Sciences, 2010, vol. 49, pp. 311-318.

\section{Сведения об авторе.}

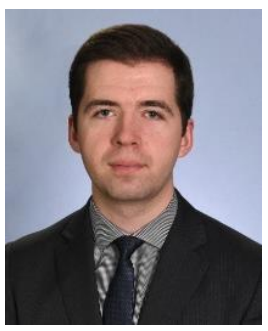

Барочкин Алексей Евгеньевич, кандидат технических наук, Ивановский государственный энергетический университет. Область научных интересов: применение матричных методов моделирования тепломассообменных процессов. E-mail: acorp27@ya.ru 